at a greater height from the observer at sea-level even when measured vertically. Thus for the distance measured in the inclined direction wo obtain :

$l=\frac{1}{\cos \delta}\left(16,000+14,620 \log \frac{1}{\cos \delta}\right) \approx \frac{\text { const. }}{\cos ^{1 \cdot 3} \delta}$

(For greater elevations such as $11 \mathrm{~km}$. use the barometric formula: $\Delta h=14,620\left(\log p_{1}-\log p_{2}\right)$ metres.)

From (4) it follows that the intensity varies in. versely as $l^{1.8}$; together with (5) wo may compute that the intensity must vary according to $\cos ^{2 \cdot 3} \delta$; the experimental result being a $\cos ^{2} \delta$ variation.

The second part of the intensity of the hard component consists of heavy particles created by neutrinos in multiple showers, the neutrinos themselves being a product of the meson decay 2 . It is, however, not quite excluded that neutrinos with magnetic moment are able to produce a coincidence with the help of two soft secondary electrons ${ }^{3}$. It can easily be seen that this part of the intensity should be invariant for every direction, since (1) the meson intensity can bo taken as uniformly distributed over all directions at the place of origin ; (2) the majority of the mesons are already disintegrated before they reach the apparatus, thus the number of the neutrinos will only slightly increase in more inclined directions through the number of those produced in further decay; and (3), on the other hand, the decrease in the number of the neutrinos in the inclined direction will also be negligible on account of their small absorption. This constituent of the intensity corresponds to the first term on the right hand side of (1).

In the case of the soft component, we can again distinguish two parts, the first consisting of electrons produced at the disintegration of the mesons, or cascade electrons created by them. This part of the intensity should vary according to the meson intensity, that is, with $\cos ^{2 \cdot 3} \delta$; but we must also take into account that the probability with which the decay occurs in the region surrounding the apparatus is inversely proportional to the energy of the meson. Thus the intensity will be expressed now by

$$
J=\text { const. } \int_{E_{1}}^{\infty} \frac{e^{-l E_{0} / r c E}}{E^{\gamma+1}} \frac{I}{E} d E \sim \frac{\text { const. }}{l \gamma+1} ;
$$

and we get finally from considerations analogous to those above that this part of the soft component should vary as $\cos ^{3 \cdot 6} \delta$. 'The experimental results are in good agreement with a variation corresponding to $\cos ^{3 \cdot 5} \delta$, the second term on the right hand side of (2).

The second constituent of the soft component furnishes again a constant intensity. It consists of electrons created by neutrinos. The same considerations as above hold also in this case; thus this part, corresponding to the first term in (2), should be invariant for all directions. We believe we have found here an explanation why the relative intensity of the soft component increases again not only for more inclined directions but also at very great depths'.

So far as the reliability of the measurements permit, we are able to estimate the amounts of the different kinds of radiation observable at sea-level from the vertical direction. 65 per cent of the radiation consists of mesons, 30 per cent of electrons produced in meson decay or cascades initiated by them, 5 per cent of electrons and heavy ionizing particles created by neutrinos.
We wish to express our gratitude to the Hungarian Council for Natural Science, and the Hungarian Academy of Science for financial support.

$$
\text { J. Barnóthy. }
$$

$$
\text { M. Forró. }
$$

Institute for Experimental Physics, University, Budapest.

$$
\text { May } 27 .
$$

${ }^{1}$ Euler, II., and Heisenberg, W., Erg.d. exact. Naturteis8., 17, 33 (1938). Yukawa, H., Proc. Phys. Math. Soc. Jap., 19, 712 (1937).

- Bethe, H., Proc. Camb. Phil. Soc., 31, 108 (1935).

- Barnóthy, J., and Forro, M., Phys. Rev., 55, 870 (1939). On 1,000 m. water-equivalent depth, about 50 per cent of the radiation con. water-equivalent dept
sists of soft particles.

\section{Effect of an Electric Field on the Viscosity of Liquids}

Prof. E. N. da C. Andrade and C. Dodd have described $^{1}$ their investigations of the effect of an electric field on the viscosity of a number of organic liquids. They state that they found an increase of viscosity of up to 60 per cent in polar liquids, while non-polar liquids showed no increased viscosity.

We would point out that work of a similar kind was performed by us in $1935^{2}$

We succeded in showing that the increase of viscosity, which in some cases was more than 100 per cent, is due to the passage of the electric current through the liquid. Increased viscosity was also observed in non-polar liquids (benzene, hexane, carbon tetrachloride) when the latter had been artificially contaminated by the addition to the pure preparations of a few drops of the same liquid saturated with hydrochloric acid vapour, or of a few drops of acetone. In our opinion, this seeming increase of viscosity in a liquid under the action of an electric field is a purely hydrodynamic phenomenon due to the convection of a non-homogeneous liquid placed in an electric field.

Physical Institute,

S. Sossinski.

Leningrad State University.

NATCRE, 143, 26 (1939).

-Acta Physirochimica U.R.S.S., 5, 691 (1936).

WE are sorry that the interesting paper of Sokolow and Sossinski, referred to in the above letter, escaped our notice, and we are very glad that our attention has now been directed to it. We agree that in it the authors clearly established a large effect of electric field on the apparent viscosity of polar liquids, and that the effect is accompanied by the passage of an electric current, both of which facts are among our observations. We think, however, that the method which Sokolow and Sossinski used, namely, a capillary of square cross-section, with the field applied to two opposite sides, is less precise than our disposition : we used a capillary of rectangular crosssection, with side ratio 1 to 50 , and the potential applied to the long sides. In our case the field is uniform; in the other case, owing to differences of dielectric constant between liquid and glass, it is far from being homogeneous, which may be one of the causes of certain discrepancies which we have noted.

Owing to the fact that the upper limit of the fields used by Sokolow and Sossinski was $17 \mathrm{kv} . / \mathrm{cm}$., while our fields went up to $40 \mathrm{kv} . / \mathrm{cm}$., the Russian workers were unable to observe the saturation effect, namely, 